\title{
CMS Reimbursement Reform
}

\author{
Risha Gidwani, DrPH ${ }^{1,2,3,4}$ and Jay Bhattacharya, MD, $P h D^{4}$ \\ IVA Palo Alto Health Care System, VA Health Economics Resource Center, Menlo Park, CA, USA; ${ }^{2}$ VA Center for Innovation to Implementation, \\ Menlo Park, CA, USA; ${ }^{3}$ Department of Medicine, Stanford University, Stanford, CA, USA; ${ }^{4}$ Centers for Health Policy, Primary Care and Outcomes \\ Research, Stanford University, Stanford, CA, USA.
}

J Gen Intern Med 30(11): 1588

DOI: $10.1007 /$ s11606-015-3465-5

(C) Society of General Internal Medicine 2015

\begin{abstract}
A uthor's response-
Dear Editor:

In our difference-in-difference-based analysis of hospital administrative data, we found a reduced incidence of pulmonary embolism (PE) and deep vein thrombosis (DVT) associated with Centers for Medicare and Medicaid Services' (CMS) payment reform for hospital-acquired conditions. In their comment, Taylor, Ecker and Taylor suggest this may be due to hospitals reducing their screening practices for this postsurgical complication. This is a possible explanation, but one which we consider unlikely. Our findings of reduced incidence of PE or DVT post-CMS reimbursement reform were due to both a decrease in complication incidence in the
\end{abstract}

Medicare population, as well as an increase in incidence of complication in the non-Medicare population. If screening was the main factor, as Taylor et al. suggest, hospitals would have to influence physicians to reduce screening in Medicare patients while also influencing physicians to increase screening in non-Medicare patients. Given the challenges often faced in changing provider practice patterns, we consider it improbable that hospitals were able to successfully persuade physicians to change their screening practices - in opposite directions based on patient insurance status - in the few months after CMS payment reform. Nevertheless, Taylor, Ecker and Taylor identify an important mechanism that could benefit from further study.

Sincerely,

Risha Gidwani and Jay Bhattacharya

Corresponding Author: Risha Gidwani, DrPH; VA Palo Alto Health Care SystemVA Health Economics Resource Center, Menlo Park, CA, USA (e-mail: risha.gidwani@va.gov).

Published online July 16, 2015 